\title{
Profesorado novel en las universidades catalanas: análisis de sus programas formativos
}

\author{
Gabriel Hervas ${ }^{1}$ (D) , José Luis Medina ${ }^{2}$
}

Artículo de investigación. Recibido: 07/01/2020. Aceptado:12/03/2020. Publicación avanzada: 04/06/2020. Publicado: 01/07/2020

Resumen

INTRODUCCIÓN. Esta investigación tiene por objetivo identificar qué universidades catalanas organizan un programa de alcance institucional destinado especialmente a la formación en docencia de su profesorado novel y examinar y comparar sus características y contenidos.

MÉTODO. Se realizó un análisis documental y de contenido de los datos recogidos sistemáticamente en documentos institucionales, estudios publicados y en las respuestas recibidas desde las instituciones a nuestras cuestiones.

RESULTADOS. Cinco de las 12 universidades catalanas organizan un programa de estas características. Entre estos, se señala la tendencia a tratar determinados contenidos - TIC, evaluación, metodologías y diseño de asignaturas-, practicar la observación en el aula y usar el portafolio como herramienta evaluativa. En cambio, contenidos como la tutoría y el trabajo en equipo son menos frecuentes, y existen claras diferencias en el diseño de los programas, horas de dedicación y origen -institucional y departamental- de sus formadores.

DISCUSIÓN. Centramos la discusión en las características, diferencias y elementos comunes observados, proponiendo ideas y argumentos por ejemplo, la inclusión de contenidos relacionados con la diversidad en el aula o la docencia en otros idiomas- que responden a lo que la literatura apunta actualmente al respecto de la formación del profesorado universitario.

Palabras clave

Profesor novel; Formación inicial; Programa de formación; Desarrollo profesional.

Datos de los autores

1 Universitat de Barcelona, España. Departamento de Didáctica y Organización Educativa. Contacto para la correspondencia: ghervas@ub.edu

${ }^{2}$ Universitat de Barcelona, España. Departamento de Didáctica y Organización Educativa.

Referencia recomendada

Hervas, G., y Medina, J. L. (2020). Profesorado novel en las universidades catalanas: análisis de sus programas formativos. REIRE Revista d'Innovació i Recerca en Educació, 13(2), 1-19. https://doi.org/10.1344/reire2020.13.230614

C 2020 Gabriel Hervas y José Luís Medina. Este artículo es de acceso abierto sujeto a la licencia Reconocimiento 4.0 Internacional de Creative Commons, la cual permite utilizar, distribuir y reproducir por cualquier medio sin restricciones siempre que se cite adecuadamente la obra original. Para ver una copia de esta licencia, visite http://creativecommons.org/licenses/by/4.0/ 
G. Hervas y J. L. Medina. Profesorado novel en las universidades catalanas: análisis de sus programas formativos

Títol

Professorat novell a les universitats catalanes: anàlisi dels seus programes formatius

Resum

INTRODUCCIÓ. Aquesta recerca té per objectiu identificar quines universitats catalanes organitzen un programa d'abast institucional especialment destinat a la formació en docència del seu professorat novell i examinar-ne i comparar-ne les característiques i els continguts.

MÈTODE. Es va fer una anàlisi documental i de contingut de les dades recollides sistemàticament en documents institucionals, en estudis publicats $i$ en les respostes rebudes des de les institucions a les nostres qüestions.

RESULTATS. Cinc de les dotze universitats catalanes organitzen un programa d'aquestes característiques. En aquests programes, s'observa la tendència a tractar determinats continguts - TIC, avaluació, metodologies i disseny d'assignatures-, practicar l'observació a l'aula i utilitzar el portafolis com a instrument d'avaluació. En canvi, continguts com la tutoria i el treball en equip són menys freqüents, i hi ha clares diferències en el disseny dels programes, les hores de dedicació i l'origen -institucional i departamental- dels seus formadors.

DISCUSSIÓ. Centrem la discussió en les característiques, diferències i elements comuns observats, i proposem idees i arguments — per exemple, incloure continguts relacionats amb la diversitat a l'aula o la docència en altres idiomes - que responen al que la literatura apunta actualment sobre la formació del professorat universitari.

Paraules clau

Professor novell; Formació inicial; Programa de formació; Desenvolupament professional.

Title

New faculty members at Catalan universities: analysis of their training programmes

Abstract

INTRODUCTION. The goal of this research is to identify which Catalan universities organize institution-wide programmes especially designed to train their new faculty members, and to examine and compare their features and content.

METHOD. We conducted a documentary and content analysis of data systematically gathered from institutional documents and published studies, and the answers to our questions received from the institutions.

RESULTS. Five out of the 12 Catalan universities organize programmes of this kind. Among them, we identify a tendency to address certain specific contents-ICT, assessment, teaching methods and course design - to organize classroom observations, and to use portfolios as an assessment tool. We also find that the presence of other content such as tutorial support and teamwork is less frequent, and note clear differences regarding programme design, the dedication required of the participants, and the institutional and departmental origin of the instructors.

DISCUSSION. We focus on the features, differences and common trends in these training programmes, proposing ideas and arguments-for example, the inclusion of content addressing students' diversity and teaching in other languages - based on the discussion in the current literature in relation to the training and professional development of university faculty members.

Keywords

Novice teachers; Initial training; Training programme; Faculty development. 


\section{Introducción}

La formación y desarrollo profesional del profesorado universitario vienen siendo elementos de preocupación desde hace décadas en España. Benedito Antolí (1983) trataba ya este tema y mencionaba las primeras iniciativas interesadas en el profesorado universitario, su formación e incluso sus competencias docentes. Décadas después, el diseño y puesta en marcha de programas para la formación del profesorado novel suponen todavía un reto para las universidades españolas (Martín Gutiérrez et al., 2014), enmarcadas en un contexto ambivalente en el que, por encima de todo, se incentiva y premia la investigación como elemento primordial para ser acreditado y avanzar en la carrera docente, pero donde a la vez se desarrolla un discurso que enfatiza cada vez más la necesidad de mejorar la calidad de la docencia (Jacob et al., 2015).

Cabe no olvidar que, en el sistema educativo español, al profesor universitario es al único al que no se le exige una formación específica relacionada con la docencia, poniendo de manifiesto la diferencia que se realiza, de manera más o menos tácita, entre lo que parece significar enseñar y aprender en la universidad, y lo que ello significa en la educación primaria y secundaria, espacios en los que para acceder a la docencia es necesario cursar un grado - primaria - o máster - secundaria - universitario. Por ello, hablar de docentes en las universidades españolas es hablar, en general, de especialistas en sus disciplinas con, en cambio, una formación pedagógica deficiente (Sánchez Moreno y Mayor Ruiz, 2006). De este modo, la formación docente del profesorado universitario sigue siendo un tema de actualidad. Esto es especialmente claro en Cataluña donde, en 2017, desde la Secretaría de Universidades e Investigación de la Generalitat catalana se impulsó el "Programa Margalida Comas i Camps" (Generalitat de Catalunya, 2017) que planteaba entre sus objetivos generales la creación de un programa interuniversitario de formación docente para el profesorado universitario (en especial, para el novel). Así, en este momento en el que se empiezan a observar movimientos que pueden generar cambios en las exigencias formativas al profesorado de las universidades catalanas, resulta necesario conocer y analizar cuál es la situación en la que se encuentran dichas instituciones y, con ello, contribuir al desarrollo de las propuestas que puedan llegar a formalizarse. En este estudio se recoge dicha necesidad y se analiza la formación del profesorado novel en las 12 universidades catalanas con dos objetivos:

1. Identificar qué universidades catalanas ofrecen un programa de formación de alcance institucional especialmente diseñado y destinado para sus docentes noveles y sin que se limite su participación.

2. Describir, comparar y analizar las características y contenidos de dichos programas.

Ambos objetivos se concretan en las siguientes preguntas de investigación:

- ¿Cuenta la universidad con un programa estructurado en docencia universitaria?

- ¿Está dicho programa especialmente diseñado/destinado para la formación de los docentes noveles y no limita su participación?

- ¿Cuáles son las características de dicho programa?

- ¿Qué contenidos se trabajan? 


\subsection{Formación del profesorado universitario español}

La formación del profesorado universitario español va ligada a la creación de los Institutos de Ciencias de la Educación (ICE), nacidos en época de la dictadura franquista a partir de dos Reales Decretos en 1969 y de la posterior Ley General de Educación de 1970 (Castillejo Brull, 1982). No obstante, pese a que la legislación atribuyó a dichos ICE la función de formar al profesorado de todo el sistema educativo -incluyendo al universitario-, más centrados en los y las maestras de las etapas escolares, el interés por la formación pedagógica del profesorado universitario fue muy débil en la década de los años setenta (De la Orden, 1977) y no hallamos experiencias regulares con núcleos de especialistas en el desarrollo profesional de dicho profesorado hasta la década de los años ochenta (Aramburuzabala Higuera et al., 2013). Si comparamos esta situación con la de un país pionero en este tema, en Estados Unidos encontramos en 1962 la primera unidad para el desarrollo profesional del profesorado universitario y, ya en los años setenta, las primeras conferencias y la primera organización que ofrecía apoyo a los encargados de organizar el desarrollo profesional de los docentes, los "faculty developers" (Sorcinelli et al., 2006, p. 6).

Las universidades cuentan hoy con una ley propia, la Ley Orgánica de Universidades de 2007 (LO $4 / 2007$, de 12 de abril), en que se reconoce la formación de los docentes como un criterio relevante a la hora de evaluar su actividad profesional y de considerar retribuciones adicionales. No obstante, bajo esa misma legislación, se otorga a las instituciones universitarias autonomía para decidir de qué manera formar a su profesorado; autonomía con la que también cuentan a la hora de decidir la posible formación en competencias docentes de sus doctorandos con contratos predoctorales (Moll, 2018), quienes representan una parte importante del colectivo de docentes noveles en las universidades. Esto ha generado una clara diversidad de propuestas que han sido atendidas por, principalmente, dos tipos de trabajos en la literatura sobre formación de los docentes en las universidades españolas ${ }^{1}$. Por un lado, textos teóricos sobre modelos y tendencias de formación y desarrollo profesional del profesorado, o con ideas, recomendaciones y propuestas para el diseño de dichos programas, especialmente, en relación con el Marco Común Europeo (Imbernón, 2016; Zabalza Beraza et al., 2014). Por otro lado, estudios contextualizados en una universidad concreta en que se analizan o evalúan un programa -o algún elemento de este- - /o sus repercusiones (Conde-Jiménez y Martín-Gutiérrez, 2016; Encinar Prat y Barrera-Corominas, 2017; Luque-Vílchez, 2018).

No obstante, no contamos con abundante literatura que analice y ofrezca una panorámica para el conjunto del estado -o de Cataluña - que ayude a visibilizar qué se hace y cuáles son las tendencias en la formación de los docentes universitarios o que, en especial, permita examinar críticamente la calidad de estos programas y ayudar a tomar decisiones al respecto. El trabajo más cercano a ofrecer una perspectiva global fue el coordinado por Amador (2012) y, en él, se reflejaba que el 83,87 \% de las 31 universidades que respondieron la encuesta - siete catalanas - ofrecían un programa de formación destinado a sus noveles. Antes de ese estudio, Pinya (2008) había elaborado un estado de la cuestión sobre los programas para la formación de docentes universitarios en que, de manera asistemática, se apuntaban actividades formativas de universidades varias como la Politécnica de Madrid, la de las Illes Balears, la de Barcelona, la Rovira i Virgili o la de Granada. Tras estos dos

1. Por cuestiones de espacio, limitamos las referencias a unos pocos estudios recientes. 
trabajos, los estudios que recientemente más se acercan al objetivo de analizar los programas para la formación de los docentes universitarios de varias instituciones universitarias españolas son los de: (1) Álvarez Castillo et al. (2017), tratando la inclusión del aprendizaje-servicio en las iniciativas formativas para el profesorado universitario de 68 instituciones; (2) da Silva y Tejada Fernández (2016), con un escueto análisis comparativo entre los objetivos para la formación de los docentes de tres universidades catalanas y tres de São Paulo, Brasil; (3) Fernández-Salinero Miguel et al. (2017), que incluye un breve análisis de programas para la integración y formación del profesorado en la Universidad Complutense de Madrid, la de Granada, la de Málaga, la de Barcelona - si bien, en este caso, las autoras analizan un programa que, en realidad, no está ligado a la integración del profesorado universitario - y la Autónoma de Barcelona; (4) Montes y Suárez (2016), analizando los programas de cinco universidades - Autónoma de Barcelona, Autónoma de Madrid, Barcelona, Granada y País Vasco-; (5) Porto Castro y Mosteiro García (2016), analizando los programas para noveles en tres universidades públicas gallegas -A Coruña, Santiago de Compostela y Vigo-; y (6) Torra Bitlloch et al. (2013), con una encuesta en que se preguntaba a las unidades formativas de las universidades públicas catalanas -a las que pertenecían los mismos autores- acerca de la formación en competencias docentes.

\subsection{Necesidades formativas del profesorado universitario novel}

Si bien una figura como la del profesor asociado desdibuja el perfil que se ha venido trazando tradicionalmente del profesorado novel, la literatura suele definir al profesorado novel como una persona joven con, en el contexto español y según los autores, hasta tres (Conde-Jiménez y MartínGutiérrez, 2016; Feixas, 2002) o cinco años de experiencia en docencia (Amador, 2012; Benedito Antolí et al., 2001). Es un docente que se aproxima a la profesión con lo que Lortie (1975) definió como aprendizaje por (fruto de la) observación y al que frecuentemente se le asignan las clases/grupos más difíciles (Benedito Antolí et al., 2001; Ingersoll y Strong, 2011). Estas condiciones a menudo acaban conduciendo a situaciones de angustia e incertidumbre y a altos grados de abandono (Clandinin et al., 2015), fruto del colapso de los ideales que se había formado al enfrentarse a la realidad del aula -el reality shock de Veenman (1984)- y de la profesión.

Las dificultades y necesidades del profesorado novel han venido siendo recogidas desde hace décadas en trabajos (empíricos) dentro del contexto universitario español. Estudios como el de Conde-Jiménez y Martín-Gutiérrez (2016) recogen la estela de trabajos que han sido referencia para esta temática, entre los que destacan los originados en la Universidad Autónoma de Barcelona (Feixas, 2002), la Autónoma de Madrid (de la Cruz Tomé, 1994), la de Barcelona (Benedito Antolí et al., 2001) y la de Sevilla (Mingorance Díaz et al., 1993). El repaso a estos trabajos y a otros más recientes (véase, por ejemplo, los de Aránega Español, 2011; Bozu e Imbernón, 2016; Estepa Murillo et al., 2005; Sánchez Moreno y Mayor Ruiz, 2006) permite vislumbrar necesidades manifestadas por los propios docentes en relación con sus alumnos - por ejemplo, su motivación-, con el dominio de la materia a enseñar, con el ejercicio docente - por ejemplo, sobre metodologías y planificación de la docencia-, con el contexto institucional - por ejemplo, limitaciones de tiempo e infraestructuras-, o con su propia vivencia personal y profesional - por ejemplo, sentimientos de falta de apoyo o dificultades a la hora de socializar-. 
Estos estudios no solo revelan determinadas necesidades que son particulares de los noveles, sino que justifican también el diseño de programas especialmente diseñados para atenderlas y ayudarles a ordenar ideas y su visión de la docencia (Amador, 2012).

\section{Método}

\subsection{Foco de la investigación}

Atendiendo a los objetivos señalados en la introducción, el foco de esta investigación son aquellos programas estructurados para la formación en docencia universitaria, y no cursos, talleres, jornadas o seminarios particulares con dicha temática, ni planes de formación o estratégicos en que se recogen ideas o líneas de actuación futuras. Igualmente, este análisis se centra en programas que tengan un alcance institucional, y no en aquellos que pudieran ser organizados de manera particular desde cada facultad, centro o departamento. Por último, no ponemos el foco sobre programas diseñados para todo el personal docente e investigador (PDI), sino en aquellos que, aun dando cabida a profesorado con experiencia, señalan específicamente a los docentes noveles como sus destinatarios especiales y no les limitan su participación.

\subsection{Muestra}

La muestra para este estudio son las 12 universidades catalanas:

- Siete públicas: Universidad Autónoma de Barcelona (UAB), Universidad de Barcelona (UB), Universidad de Girona (UdG), Universidad de Lleida (UdL), Universidad Politécnica de Cataluña (UPC), Universidad Pompeu Fabra (UPF) y Universidad Rovira i Virgili (URV).

- Cinco privadas: Universidad Abat Oliba-CEU (UAO-CEU), Universidad de Vic-Universidad Central de Cataluña (UVic-UCC), Universidad Internacional de Cataluña (UIC), Universidad Ramon Llull (URL) y Universitat Oberta de Catalunya (UOC)².

\subsection{Diseño y recogida y análisis de datos}

La investigación se ha realizado desde una aproximación metodológica de carácter cualitativo. Bajo este enfoque y atendiendo a los objetivos de la investigación, la recogida de estos datos se realizó a través de las siguientes estrategias:

- Búsqueda y revisión sistemática de fuentes documentales institucionales que aportaran información sobre los programas para la formación de noveles de las universidades catalanas. A través de dicho proceso, se recogieron datos provenientes de: portales de transparencia,

2. El caso de la UOC presenta particularidades. De acuerdo con su estatuto (FUOC, s. f.) y con los datos públicos del Registro de Universidades, Centros y Títulos del Ministerio de Ciencia, Innovación y Universidades, se trata de una universidad de naturaleza privada. No obstante, por su condición de no presencial que complementa al resto de instituciones del sistema universitario catalán, a menudo no es considerada como tal. Así, pertenece, a la Asociación Catalana de Universidades Públicas y la Generalitat, aunque no la incluye entre las públicas, también la distingue de las privadas, calificándola como "no presencial" (Generalitat de Catalunya, s. f., párr. 1). 
documentos sobre la estrategia docente institucional, documentos de evaluación de la docencia, planes de calidad para la docencia, planes estratégicos, panfletos y noticias informativas institucionales y, sobre todo, de las últimas memorias institucionales, memorias de actividades de los ICE - o equivalentes - y de los propios planes/programas docentes de cada uno de los programas.

- Búsqueda y revisión de publicaciones recientes - últimos cinco años- tratando dichos programas. Se realizó la búsqueda entre las publicaciones editadas por las propias universidades y en ERIC y Google Scholar, mediante la combinación - a través de los operadores booleanos "OR" y "AND" - de conceptos clave como "formación profesorado novel", "formación docente novel", "universidad", "universitario", "programa" y "curso".

- Además, paralelamente, se contactó con las unidades responsables de la formación en cada una de las doce universidades, con el objetivo de que, si lo deseaban, complementaran los datos recogidos - que, no obstante, ya provenían de las propias universidades - y matizaran dudas que habían emergido durante el análisis - convirtiéndose así en una suerte de entrevista escrita de carácter semiestructurado-. Para ello, los autores contactaron por correo electrónico con: Unidad Técnica de Calidad (UAO-CEU), Vicerrectorado de Política Académica (URL), Unidad de Evaluación, Formación e Innovación Docente (UIC), ICE e ICE docencia (UdG), Formación del Profesorado Universitario y Vicerrectorado de Calidad e Innovación Docente (UdL), ICE, su dirección y responsable de formación del profesorado e innovación docente (URV), ICE (UPC), eLearn Center (UOC), dirección y responsables de docencia e innovación y de formación permanente del Centro de Innovación y Formación en Educación (UVIC-UCC), Centro para la Innovación en Aprendizaje y Conocimiento (UPF), Instituto de Desarrollo Profesional-ICE (UB), y Oficina de Calidad de la Docencia (UAB) ${ }^{3}$.

Los datos recogidos por estas vías - en especial, aquellos referentes a las características y contenidos de los programas - fueron analizados y comparados combinando el análisis documental (Petty et al., 2012) y de contenido (Krippendorff, 1989) de tipo convencional (Hsieh y Shannon, 2005), estrategias metodológicas para la recogida y análisis de datos interpretativos en las que los propios investigadores son considerados el principal instrumento (Eisner, 1998; Gimeno-Sacristán y Pérez, 2002) y que posibilitaron la comparación continua de los datos recogidos a través de la búsqueda de documentos y el cuestionamiento -más que entrevista- a los responsables de la formación en las instituciones universitarias analizadas.

\section{Resultados}

En relación con la primera pregunta de investigación, los datos revelan que ocho de las doce universidades catalanas $(66,67 \%)$ organizan un programa de alcance institucional para la formación en docencia universitaria. No obstante, en solo cinco de los casos $(41,67 \%)$ dicho programa está diseñado o destinado especialmente al profesorado novel (ver Tabla 1).

3. Tras contactar con ellas, seis instituciones - UB, UdL, UIC, UPC, UPF y URL - facilitaron información adicional. 
Tabla 1

Universidades con programa en docencia universitaria y diseñado/destinado especialmente al profesorado novel

\begin{tabular}{|c|c|c|c|c|c|c|c|c|c|c|c|c|}
\hline & $\begin{array}{l}\text { UAO- } \\
\text { CEU }\end{array}$ & $\begin{array}{l}\text { UVic- } \\
\text { UCC }\end{array}$ & UIC & URL & UOC & UAB & UB & UdG & UdL & UPC & UPF & URV \\
\hline PDU & No & No & Sí & No & Sí & Sí & Sí & No & Sí & Sí & Sí & Sí \\
\hline DDN & - & - & Sí & - & Sí & Sí & Sí & - & No & No & Sí & No \\
\hline
\end{tabular}

Nota. PDU: Programa en docencia universitaria. DDN: Destinado en especial al docente novel y sin limitar su participación

El análisis de la situación en las universidades privadas muestra que tres de las cinco -UAO-CEU, UVic-UCC y URL ${ }^{4}$ - no organizan una propuesta formativa específica y de alcance institucional para el profesorado novel, si bien las tres indican la existencia de una unidad o centro - la Unidad Técnica de Calidad, en la UAB-CEU, la Unidad de Calidad e Innovación Académico-Docente, en la URL, y el Centro de Innovación y Formación en Educación, en la UVic-UCC - desde los que impulsan y gestionan programas y proyectos para la calidad y la innovación en la docencia.

En lo que respecta a las universidades públicas, cuatro de las siete - UdG, UdL, UPC y URV- no ofrecen un programa que sea específico para sus noveles. No obstante, UdL, UPC y URV sí disponen de un programa estructurado en docencia universitaria abierto a todo el PDI, incluido el profesorado novel. Sin embargo, en ninguno de estos programas se les señala como destinatarios especiales. No solo eso, sí que se apuntan destinatarios de interés alejados de la figura del profesorado novel — tal es el caso, por ejemplo, de la UdL y su mención especial a docentes que estuvieran desarrollando nuevas titulaciones - o se limita la participación de determinados contratados predoctorales - con posibilidad de ejercer docencia y, por tanto, noveles - a solo algunos cursos - caso de la UPC $-{ }^{5}$. De cualquier modo, dichos programas merecen ser destacados ya que demandan a sus participantes una dedicación notable: 15 créditos ECTS en la UPC alrededor de $375 \mathrm{~h}-, 30$ ECTS en la URV $-750 \mathrm{~h}$ - y entre 58 y 90,5 h en la UdL -en función de los cursos optativos seleccionados-. Por otro lado, en cuanto a la UdG, si bien no en el presente, hasta 2014-2015 organizaba un curso de postgrado en docencia universitaria especialmente dirigido a los noveles. Algo similar sucedía en la UPC donde, antes del postgrado actual abierto a todo el PDI, existía un programa de formación inicial para el PDI de nueva incorporación.

Entre las universidades que sí organizan un programa especialmente destinado al profesorado novel y de alcance institucional, las características de dichos programas -en su última edición- se resumen en las tablas $2 a$ y $2 b$.

4. En el caso de la URL, no obstante, desde el Vicerrectorado de Política Académica se apunta que, dada la naturaleza de la institución, más allá de una acogida inicial en el rectorado, la formación del profesorado novel la gestiona cada facultad/institución con el propósito de ajustarla a las necesidades formativas específicas. De este modo, no tratándose de formaciones de alcance institucional, quedan fuera del objeto de esta investigación.

5. Desde la dirección del programa de la UPC se señala que se está estudiando cómo conseguir que estos contratados predoctorales puedan participar en todos los cursos. Por ahora, esto no es posible dadas las condiciones para la subvención de algunos cursos. 
G. Hervas y J. L. Medina. Profesorado novel en las universidades catalanas: análisis de sus programas formativos

Tabla 2a

¿Cuáles son las características de los programas formativos específicos para la formación de los docentes noveles?

\begin{tabular}{|c|c|c|c|c|}
\hline UIC & UOC & UAB & UB & UPF \\
\hline \multicolumn{5}{|c|}{ Programa } \\
\hline $\begin{array}{l}\text { Programa Gaudí de } \\
\text { Formación del } \\
\text { profesorado }\end{array}$ & $\begin{array}{l}\text { Formación básica para la } \\
\text { docencia en la UOC (eLC } \\
\text { License) }\end{array}$ & $\begin{array}{l}\text { Programa de acreditación } \\
\text { Formación docente en } \\
\text { educación superior }\end{array}$ & $\begin{array}{c}\text { Máster en Docencia } \\
\text { universitaria para } \\
\text { profesorado novel }\end{array}$ & $\begin{array}{c}\text { Certificado de Formación } \\
\text { inicial en docencia } \\
\text { universitaria } \\
\text { (itinerario A) }\end{array}$ \\
\hline \multicolumn{5}{|c|}{ Duración, ECTS y horas de dedicación } \\
\hline 1 curso & 3 meses & 1.5 cursos & 2 cursos & 1 curso \\
\hline 3 ECTS & 2 ECTS & 7 ECTS & 60 ECTS & 7 ECTS \\
\hline $75 \mathrm{~h}$ & $50 \mathrm{~h}$ & $175 \mathrm{~h}$ & $1500 \mathrm{~h}$ & $170 \mathrm{~h}$ \\
\hline \multicolumn{5}{|c|}{ Destinatarios y experiencia docente } \\
\hline $\begin{array}{l}5 \text { o menos años de } \\
\text { experiencia docente }\end{array}$ & $\begin{array}{l}\text { Profesorado propio de } \\
\text { nueva incorporación }\end{array}$ & $\begin{array}{l}\text { Preferentemente, menos } \\
\text { de } 5 \text { años en la UAB }\end{array}$ & $\begin{array}{l}\text { No más de } 5 \text { años de } \\
\text { experiencia docente }\end{array}$ & $\begin{array}{c}\text { PDI de nueva } \\
\text { incorporación en la UPF o } \\
\text { con menos de } 3 \text { años de } \\
\text { docencia en ella }\end{array}$ \\
\hline \multicolumn{5}{|c|}{ Límite de participantes } \\
\hline No se indica & 30 & 40 & 30 & 20 \\
\hline \multicolumn{5}{|c|}{ Modalidad de formación } \\
\hline Semipresencial & Virtual & Presencial & $\begin{array}{c}\text { Eminentemente } \\
\text { presencial (posibilidad de } \\
\text { algún módulo optativo o } \\
\text { contenido particular } \\
\text { virtual) }\end{array}$ & Semipresencial \\
\hline \multicolumn{5}{|c|}{ Mentoría } \\
\hline Sí & Al margen del programa & No & Sí & No \\
\hline \multicolumn{5}{|c|}{ Evaluación } \\
\hline $\begin{array}{l}\text { Asistencia y portafolio } \\
\text { con actividades del curso }\end{array}$ & $\begin{array}{l}\text { La información disponible } \\
\text { no permite responder } \\
\text { (n.d.) }\end{array}$ & Actividades y portafolio & $\begin{array}{l}\text { Asistencia, actividades en } \\
\text { cada módulo, portafolio y } \\
\text { presentación portafolio }\end{array}$ & $\begin{array}{c}\text { Asistencia, actividades } \\
\text { online, trabajo individual } \\
\text { y portafolio }\end{array}$ \\
\hline
\end{tabular}

Tabla 2b

¿Cuáles son las características de los programas formativos especificos para la formación de los docentes noveles? (continuación)

\begin{tabular}{|c|c|c|c|c|}
\hline UIC & UOC & UAB & UB & UPF \\
\hline \multicolumn{5}{|c|}{ Número de formadores de módulos obligatorios } \\
\hline 15 & n.d. & 7 & 23 & 8 \\
\hline \multicolumn{5}{|c|}{ Origen institucional de los formadores } \\
\hline UIC (n=14; $93.33 \%)$ & n.d. & $\mathrm{UAB}(\mathrm{n}=4 ; 57.14 \%)$ & $\begin{array}{c}\text { UB PDI } \\
(n=14 ; 60.87 \%)\end{array}$ & UPF $(n=3 ; 37.5 \%)$ \\
\hline \multirow{17}{*}{$\begin{array}{c}\text { Otros: } \\
\text { Externo sin concretar } \\
(n=1 ; 6.67 \%)\end{array}$} & & Otros: & UB técnicas de & Otros: \\
\hline & & UPC ( $n=2 ; 28.57 \%)$ & formación IDP-ICE (n=2; & No universitario $(n=3$; \\
\hline & & UB $(n=1 ; 14.29 \%)$ & $8.70 \%)$ & $37.5 \%)$ \\
\hline & & & UB emérito $(n=1 ; 4.35 \%)$ & UAB (n=1; $12.5 \%)$ \\
\hline & & & UB Centro Recursos para & UAB/University of \\
\hline & & & el Aprendizaje y la & Teacher Education Zurich \\
\hline & & & $\begin{array}{l}\text { Investigación ( } \mathrm{n}=1 ; \\
4.35 \%)\end{array}$ & \\
\hline & & & [Total UB=18; $78.26 \%$ ] & \\
\hline & & & Otros: & \\
\hline & & & No universitario $(n=2$; & \\
\hline & & & $8.70 \%)$ & \\
\hline & & & UIC (n=1; $4.35 \%)$ & \\
\hline & & & Univ. Politécnica de & \\
\hline & & & Valencia emérito $(n=1$; & \\
\hline & & & $4.35 \%))$ & \\
\hline & & & Univ. Iberoamericana & \\
\hline & & & $(n=1 ; 4.35 \%)$ & \\
\hline
\end{tabular}


G. Hervas y J. L. Medina. Profesorado novel en las universidades catalanas: análisis de sus programas formativos

\begin{tabular}{|c|c|c|c|c|}
\hline UIC & UOC & UAB & UB & UPF \\
\hline \multicolumn{5}{|c|}{ Departamento al que pertenecen los formadores adscritos a un centro universitario } \\
\hline n.d. & n.d. & $\begin{array}{l}\text { Didácticas generales y } \\
\text { específicas }(n=4) \\
\text { Filología catalana }(n=1) \\
\text { Arquitectura de } \\
\text { computadores }(n=1) \\
\text { Ingeniería civil y } \\
\text { ambiental }(n=1)\end{array}$ & $\begin{array}{c}\text { Didáctica y organización } \\
\text { educativa ( } n=4) \\
\text { Educación lingüística y } \\
\text { literaria, y didáctica de } \\
\text { las ciencias } \\
\text { experimentales y de la } \\
\text { matemática ( } n=4) \\
\text { Biología celular, fisiología } \\
\text { e inmunología ( } n=2 \text { ) } \\
\text { Teoría e historia de la } \\
\text { educación ( } n=2 \text { ) } \\
\text { Educación ( } n=1 \text { ) } \\
\text { Métodos de } \\
\text { investigación y } \\
\text { diagnóstico en educación } \\
\text { ( } n=1 \text { ) } \\
\text { Enseñanza y aprendizaje } \\
\text { mediados por } \\
\text { tecnologías ( } n=1 \text { ) } \\
\text { Personalidad, Evaluación } \\
\text { y Tratamiento } \\
\text { Psicológico ( } n=1 \text { ) }\end{array}$ & $\begin{array}{l}\text { Didácticas generales } \\
\qquad(\mathrm{n}=2) \\
\text { Ciencias experimentales } \\
\text { y de la salud }(\mathrm{n}=1) \\
\text { Ciencias políticas y } \\
\text { sociales }(\mathrm{n}=1) \\
\text { Tecnologías de la } \\
\text { información y las } \\
\text { comunicacio-nes }(n=1)\end{array}$ \\
\hline
\end{tabular}

Por otro lado, los contenidos que se trabajan en los diferentes programas tienen que ver con los que aparecen en la Tabla 3.

\section{Tabla 3}

¿Con qué guardan relación los contenidos de los programas?

\begin{tabular}{|c|c|c|c|c|c|}
\hline & UIC & UOC & UAB & UB & UPF \\
\hline Evaluación del aprendizaje & Sí & Sí & Sí & Sí & Sí \\
\hline Procesos de enseñanza-aprendizaje & No & Sí & No & Sí & Sí \\
\hline Trabajo en equipos docentes & No & Sí & No & Sí & No \\
\hline Comunicación & Sí & No & Sí & Sí & Sí \\
\hline Metodologías docentes & Sí & No & Sí & Sí & Sí \\
\hline Nuevas tecnologías (TIC) y docencia & Sí & Sí & Sí & Sí & Sí \\
\hline $\begin{array}{l}\text { Planificación de curso y/o diseño de } \\
\text { asignaturas }\end{array}$ & Sí & Sí & Sí & Sí & Sí \\
\hline Creación de materiales y recursos docentes & No & Sí & Sí & Sí & No \\
\hline Tutoría y/o plan de acción tutorial & No & No & No & Sí & Sí \\
\hline $\begin{array}{l}\text { Misión de las instituciones universitarias y } \\
\text { Espacio Europeo de Educación Superior } \\
\text { (EEES) }\end{array}$ & Sí & n.d. & Sí & Sí & No \\
\hline Ética y valores profesionales & Sí & No & No & Sí & Sí \\
\hline Perspectiva de género en la docencia & No & No & No & No & Sí \\
\hline $\begin{array}{l}\text { Investigación y transferencia del } \\
\text { conocimiento }\end{array}$ & Sí & No & No & No & No \\
\hline Observación en el aula & Sí & No & Sí & Sí & Sí \\
\hline Procesos de acreditación docente & Sí & No & No & No & No \\
\hline La universidad y su modelo educativo & No & Sí & No & Sí & Sí \\
\hline
\end{tabular}


Finalmente, los datos analizados permiten complementar los resultados presentados en las tablas 2 y 3 tal y como sigue:

- UIC: se trata de un programa cuyos contenidos y formadores se ajustan en función de las necesidades puntuales de cada centro. En las tres ediciones hasta ahora celebradas han participado, respectivamente, 26 docentes de odontología, 11 de ciencias sociales y 22 de ciencias de la salud.

- UOC: además del programa eLC License iniciado en 2016, la universidad ofrece otros dos programas para docentes que empiezan en la universidad - uno para profesorado de titulaciones interuniversitarias y otro para profesores colaboradores-. La dedicación y los contenidos de ambos programas son más limitados ya que son de 0,5 y 1 ECTS respectivamente y, principalmente, se centran en presentar el modelo educativo y de docencia de la universidad, su soporte a la docencia, las herramientas disponibles y el campus virtual y el diseño de asignaturas en él.

Por otro lado, en cuanto al programa aquí analizado, si bien no incluye mentoría, la UOC ofrece a su profesorado propio un programa paralelo de mentoring para ayudarle en su incorporación a la docencia.

- UAB: se ha detectado que uno de sus formadores participa también como formador en el programa de la UB.

- UB: además del programa analizado, la universidad ofrece un curso presencial de $14 \mathrm{~h}$ lectivas para profesorado de nueva incorporación en que se tratan roles del profesorado, estilos de aprendizaje, planificación y evaluación, comunicación en el aula y recursos y normas de la universidad.

- UPF: se trata de un programa destinado especialmente al profesorado novel que, no obstante, abre la posibilidad de participar a profesorado con más de tres años de experiencia si cuentan con plazas disponibles. El programa cuenta con otros dos itinerarios alternativos $-\mathrm{B}$ y $\mathrm{C}-$, más limitados en cuanto a la dedicación -3 y 1 ECTS respectivamente- y a la no inclusión de tutorías pedagógicas -sí, en cambio, sobre la observación-. El itinerario $C$, además, es en inglés y virtual. Finalmente, se ha detectado un formador que participa también en el programa de la UB.

\section{Discusión y conclusión}

La existencia de un programa de alcance institucional para la formación en docencia del profesorado de la mayoría de las 12 universidades catalanas - UAB, UB, UdL, UIC, UOC, UPC, UPF y URV - revela que se interpreta como una necesidad el desarrollo de un tipo de formación estructurada y organizada que vaya más allá de cursos, seminarios, talleres o jornadas particulares.

No obstante, esta interpretación no se ve reflejada de igual forma en las universidades privadas. Entre estas cinco, solo dos - UIC y UOC - ofrecen evidencias de la realización de un programa - no cursos o jornadas particulares- de alcance institucional en docencia para su profesorado, sea novel o no. Como se ha apuntaba en los resultados, cabe la posibilidad de que en UAO-CEU y UVic-UCC suceda como en la URL y que, pese a contar con unidades y centros desde los que, en principio, se impulsan y coordinan las iniciativas de formación e innovación en docencia universitaria a nivel institucional, se derive la responsabilidad y 
diseño de la formación a las respectivas facultades, centros o departamentos. Esta manera de hacer común en el albor de los programas de desarrollo profesional del profesorado en Estados Unidos (Gaff y Simpson, 1994), aunque paulatinamente fue sustituida por la gestión desde centros institucionales- puede tener su justificación en el carácter particular de dichas universidades privadas. En cualquier caso, si bien derivar el diseño de los programas a las diferentes facultades o departamentos puede tener efectos positivos cuando contribuye a contextualizar la formación (Clarke y Hollingsworth, 2002), esto a la vez los invisibiliza y dificulta su revisión, análisis y posible evaluación externa. Así, otra estrategia para contextualizar la formación - aun partiendo de un diseño y desarrollo para toda la institución - es la que se ha hallado en UIC, UOC, UB y UPF. La primera plantea un programa para toda la institución, pero ajustable a las necesidades de cada facultad/disciplina. Las otras tres concretan o complementan el programa en docencia para sus noveles con itinerarios alternativos (UPF), programas según el perfil (UOC) y un curso de duración más limitada (UB), ofreciendo así mayor variedad para facilitar el acceso de los noveles a la formación y responder de manera más concreta a sus necesidades formativas.

En cuanto a las universidades públicas, pese a que seis de las siete organizan un programa sobre docencia universitaria, solo tres - UAB, UB y UPF- los han diseñado para los noveles o mencionan a estos como destinatarios especiales. Una situación distinta a la reflejada en Amador (2012) ya que, si bien allí no se señalaba la situación concreta de cada universidad, se ha puesto de manifiesto aquí que dos universidades que participaron en el estudio y entonces contaban con un programa especialmente destinado a la formación de docentes noveles - UdG y UPC-, ahora ya no lo hacen o no lo destinan especialmente a este profesorado. En este sentido, emerge la cuestión acerca cómo puede haber influido la situación económica que, durante años, ha dificultado la contratación de nuevo profesorado universitario a la hora de valorar la necesidad de estos programas.

En todo caso, que los programas de las otras tres universidades - UdL, UPC y URV - estén destinados a todo su profesorado, no los invalida como iniciativas útiles, también, para la formación de los noveles; de hecho, las propuestas formativas de UPC y URV exigen a sus participantes una dedicación mayor que cualquier otra de las analizados, a excepción de la de la UB. No obstante, el que no se hayan diseñado o destinado especialmente a los noveles - pese a los estudios que, mencionados anteriormente, justifican el diseño de formaciones específicas - puede limitar su potencial a la hora de responder a las características y necesidades concretas de estos, dejándolos también fuera del foco de nuestro análisis dados los objetivos de este trabajo.

Así, son cinco de las doce universidades catalanas - UIC, UOC, UAB, UB y UPF- las que organizan un programa especialmente diseñado o destinado para la formación inicial de su profesorado, lo que revela un incremento en relación con el "menos de un 25 \%" que se apuntaba en Torra Bitlloch et al. (2013, p. 290). Es pertinente señalar la disparidad existente entre ellas -en cuanto a su naturaleza, número de alumnos, titulaciones, PDI, etc.-, sobre todo en los casos de UIC y UOC. Este contraste y la autonomía de la que disponen las universidades se combinan para que no hallemos una modalidad formativa predominante - si bien la incorporación de módulos o actividades virtuales está presente en la mayoría de las iniciativas - y para que se observe que solicitan a sus participantes una dedicación desigual, dificultando así la comparación entre los programas, particularmente en el caso de la UB, con una propuesta que exige una dedicación casi diez veces superior a cualquiera de las otras. 
Como ya encontrábamos en Amador (2012), es mayoritaria la opción de situar a los potenciales destinatarios de los programas en sus cinco primeros años de docencia. De este modo, se caracteriza al novel de manera similar a como lo hacían Benedito Antolí et al. (2001), pero se incluye a profesorado que puede estar viviendo momentos diferenciados - titubeo o consolidación- en relación con su aproximación a la docencia (Huberman, 1990). No obstante, si bien mayoritaria, sigue sin contarse con una comprensión unánime de lo que es ser novel en cuanto a sus años de experiencia; una situación extensible a otras regiones del estado como reflejan, por ejemplo, los cuatro años de experiencia docente máxima para el programa de la Universidad de A Coruña (Porto Castro y Mosteiro García, 2016). Además, mientras en el caso de la UAB y la UPF se refieren a los años de experiencia en la propia universidad, en el resto de los programas no se ha realizado dicha concreción. Así, a la hora de valorar y considerar lo que significa ser novel, al elemento temporal, se le añade también un elemento contextual, relacionado con su experiencia en una u otra institución.

Por otro lado, la figura del mentor, considerada crucial $-\mathrm{y}$ sostenible- para atender necesidades formativas y dificultades individuales (Jacob et al., 2015) durante el proceso de aclimatación de los noveles, sigue sin aparecer en todos los programas. En cambio, es corriente el uso del portafolio como herramienta evaluativa, dado su potencial para fomentar, y dar visibilidad a, la reflexión (Körkö et al., 2016). Es común también la práctica de la observación en el aula, una estrategia que contribuye al desarrollo de las habilidades profesionales del profesorado (Steinert, 2010). No obstante, es necesario incidir en que este estudio se señala la inclusión o no de estos elementos, pero no se trata cómo se ponen en práctica desde un punto de vista pedagógico, una limitación que se invita a superar en futuros trabajos.

En nuestros resultados se ha puesto también de manifiesto que el origen del profesorado es institucionalmente más variado en UAB y UPF que en UB y UIC, y solo se han detectado dos formadores que participan en el programa para la formación de noveles de más de una institución. Pese a que determinadas instituciones puedan dar respuesta interna a su necesidad de formadores, una mayor colaboración con formadores de otras universidades puede contribuir a desarrollar una visión común y más global a la hora de diseñar estos programas. Por otro lado, se ha recogido una relevante presencia de profesorado con una adscripción a un departamento no relacionado con la educación, en especial en UAB y UPF; profesorado que, no obstante, suele pertenecer a grupos de innovación docente, ejerce su tarea en Ios ICE o es reconocido por su trabajo en relación con la docencia. Pese a ello, si se argumenta que los docentes universitarios necesitan formación ya que su vacío formativo es difícilmente subsanable con solo experiencia y práctica (Amador, 2012), es difícil no trasladar también este argumento a sus formadores. De otro modo, puede creerse que casi cualquiera puede llegar a ejercer dicha función (Harland y Staniforth, 2008). Experiencia y práctica - elementos, también, pero no solo, de primordial importancia- no siempre podrán cubrir el vacío que deja la falta de una formación apropiada - y entiéndase como apropiada aquella, al menos, del tipo y calidad que exigimos a los profesores en primaria y secundaria-.

Por último, se han evidenciado el trabajo de unos contenidos no muy alejados entre sí. Entre estos, destaca la formación en evaluación de los aprendizajes y en TIC, únicos contenidos presentes de manera clara en los cinco programas. En cuanto a la primera, su presencia busca dar respuesta a uno de los retos más importantes a los que se enfrenta el profesorado (Sorcinelli, 2007). En cuanto a la segunda, su inclusión debiera ser analizada en profundidad ya que es necesario ir más allá del, como señalaba Cabero Almenara (2014, p. 113), "audividualismo" y de la tendencia común a basar la formación en mejorar en el uso de instrumentos. Otros ejes fundamentales en dichos programas responden a necesidades manifestadas por 
los noveles y tienen que ver con la evaluación, la innovación en metodologías docentes y la planificación y el diseño de asignaturas. En el lado contrario, se sitúan contenidos que reciben una atención minoritaria; entre ellos, encontramos la tutoría, dimensión destacada en el modelo del EEES e importante en la formación académica del alumnado (Álvarez Pérez, 2013), y el trabajo en equipos docentes, clave por ser junto a la colaboración una dificultad a la que se refieren los docentes noveles (Feixas, 2002). Lo mismo sucede con la formación en procesos de acreditación docente, un elemento más conectado con la profesión docente que con la docencia, pero que es una preocupación fundamental para el profesor universitario novel dada su precaria situación laboral (Torra Bitlloch et al., 2013). Finalmente, cabe esperar que los programas empiecen a atender con mayor énfasis - como parte de sus contenidos, y no en cursos alternativos - a la creciente diversidad entre los estudiantes (Austin y Sorcinelli, 2013), a la internacionalización y la docencia en un idioma extranjero y, como ya sucede en el programa de la UPF, a la perspectiva de género.

Concluyendo, este trabajo aporta una fotografía de la situación actual en cuanto a la formación del profesorado universitario novel catalán; no obstante, esto no debiera ser más que una base desde la que profundizar en cómo se desarrollan estos programas. Cuarenta años atrás, De la Orden (1977) caracterizaba la situación en España de la formación del profesorado de enseñanza media como una multitud de cursos con la pretensión de enseñar aquello que es novedoso en educación sin el aval de una evaluación aceptable. Hoy, esta descripción puede definir la situación de la formación pedagógica inicial $-y$ continua - del profesorado universitario. Así, por más que se hayan desarrollado protocolos para la evaluación de estos programas (Zabalza Beraza, 2011) o que, en ocasiones, se cuente con sistemas internos de garantía de calidad (Pagès Costas et al., 2016), sus contenidos y cualidades todavía necesitan ser acreditados externamente (Aramburuzabala Higuera et al., 2013). De cara a estudios futuros, dado que una gran parte de los que disponemos actualmente tienen su origen y autoría en los propios centros y formadores que diseñan estos programas, debiera incrementarse el número trabajos desde una perspectiva externa y, como solicitan Chalmers y Gardiner (2015), hacerlo desde una posición evaluativa, partiendo de propuestas como la de Fink (2013) y recogiendo evidencias desde su planificación y a través de la observación de su implementación para, con ello, ayudar a crear un marco referencial que permita diseñar los programas a partir de lo aprendido en ellos (Feixas et al., 2015).

Organismos colaboradores: Ministerio de Economía y Competitividad (EDU2015-63712-P - BES-2016076824).

\section{Referencias}

Álvarez Castillo, J. L., Martínez Usarralde, M. J., González González, H., y Buenestado Fernández, M. (2017). El aprendizaje-servicio en la formación del profesorado de las universidades españolas. Revista Española de Pedagogía, 75(267), 199-217. https://doi.org/10.22550/REP75-2-2017-02

Álvarez Pérez, P. R. (2013). La función tutorial del profesorado universitario: una nueva competencia de la labor docente en el contexto del EEES. Revista portuguesa de pedagogía, 47(2), 85-106. https://doi.org/10.14195/1647-8614_47-2_5

Amador, J. A. (coord.) (2012). La formación del profesorado novel en la Universidad de Barcelona. Octaedro. 
Aramburuzabala Higuera, P., Martínez-Garrido, C., y García-Peinado, R. (2013). La formación del profesorado universitario en España: evolución y perspectivas. Educación, 22(43), 7-25. http://revistas.pucp.edu.pe/index.php/educacion/article/view/7494/7733

Aránega Español, S. (2011). Detecció i anàlisi de les necessitats formatives del docent universitari. Un estudi de casos múltiple [Tesis doctoral, Universitat de Barcelona]. https://www.tesisenred.net/handle/10803/52865

Austin, A. E., y Sorcinelli, M. D. (2013). The Future of Faculty Development: Where Are We Going? New Directions for Teaching and Learning, 133, 85-97. https://doi.org/10.1002/tl.20048

Benedito Antolí, V. (1983). La docencia en la universidad. Cualidades, formación y evaluación el profesorado universitario. REIS: Revista Española de Investigaciones Sociológicas, 24(83), 143-161. http://www.reis.cis.es/REIS/PDF/REIS_024_07.pdf

Benedito Antolí, V., Imbernón Muñoz, F., y Félez Rodríguez, B. (2001). Necesidades y propuestas de formación del profesorado novel de la Universidad de Barcelona. Profesorado: Revista de curriculum y formación del profesorado, 5(2), 75-102. https://www.redalyc.org/pdf/567/56750205.pdf

Bozu, Z., e Imbernón Muñoz, F. (2016). La formación docente en momentos de cambios: ¿Qué nos dicen los profesores principiantes universitarios? Profesorado, Revista de Currículum y Formación del Profesorado, 20(3), 467-492. https://recyt.fecyt.es/index.php/profesorado/article/view/54607/33236

Cabero Almenara, J. (2014). Formación del profesorado universitario en TIC. Aplicación del método DELPHI para la selección de los contenidos formativos. Educación XX1, 17(1), 111-132. https://doi.org/10.5944/educxx1.17.1.10707

Castillejo Brull, J. L. (1982). Los ICEs y la formación del profesorado. Revista de educación, 269, 43-54. http://www.educacionyfp.gob.es/dam/jcr:4b3e81af-8daa-4a56-83c897a0065f1243/re2690313059-pdf.pdf

Chalmers, D., y Gardiner, D. (2015). The measurement and impact of university teacher development programs. Educar, 51(1), 53-80. https://doi.org/10.5565/rev/educar.655

Clandinin, D. J., Long, J., Schaefer, L., Downey, C. A., Steeves, P., Pinnegar, E., McKenzie, S., y Wnuk, S. (2015). Early career teacher attrition: intentions of teachers beginning. Teaching Education, 26(1), 1-16. https://doi.org/10.1080/10476210.2014.996746

Clarke, D., y Hollingsworth, H. (2002). Elaborating a model of teacher professional growth. Teaching and Teacher Education, 18(8), 947-967. https://doi.org/10.1016/S0742-051X(02)00053-7

Conde-Jiménez, J., y Martín-Gutiérrez, A. (2016). Potencialidades y necesidades de mejora en la formación de profesores noveles universitarios. REDIE Revista Electrónica de Investigación Educativa, 18(1), 140-152. http://redie.uabc.mx/redie/article/view/767 
da Silva, L. L., y Tejada Fernández, J. (2016). La formación del profesorado universitario en Cataluña y Sao Paulo: dilemas y desafíos. Revista Española de Educación Comparada, 27, 193-213. https://doi.org/10.5944/reec.27.2016.15989

de la Cruz Tomé, M.Á. (1994). Formación inicial del profesor universitario: fundamentación teórica y experiencias en la Universidad Autónoma de Madrid. Revista de enseñanza universitaria, 7(8), 1134. http://hdl.handle.net/11441/53765

De la Orden, A. (1977). Hacia el desarrollo de competencias docentes específicas. Una experiencia en la formación pedagógica de profesores. Revista española de pedagogía, 35(138), 349-413. https://revistadepedagogia.org/wp-content/uploads/2018/04/1-Hacia-el-Desarrollo-deCompetencias-Docentes-Especificas.pdf

Eisner, E. W. (1998). El ojo ilustrado. Indagación cualitativa y mejora de la práctica educativa. Paidós.

Encinar Prat, L., y Barrera-Corominas, A. (2017). La transferència de la formació del professorat universitari: anàlisi del disseny del Programa de Formació Inicial (UPF). Temps d'Educació, 52, 205-223. https://www.raco.cat/index.php/TempsEducacio/article/view/328526

Estepa Murillo, P., Mayor Ruiz, C., Hernández, E., Sánchez, M., Rodríguez, J. M., Altopiedi, M., y Torres, J. J. (2005). Las necesidades formativas docentes de los profesores universitarios. Revista Fuentes, 6. https://revistascientificas.us.es/index.php/fuentes/article/view/2393

Feixas, M. (2002). El profesorado novel: Estudio de su problemática en la Universitat Autónoma de Barcelona. Revista de docencia universitaria, 2(1), 33-44. https://revistas.um.es/redu/article/view/11821/0

Feixas, M., Lagos, P., Fernández, I., y Sabaté, S. (2015). Modelos y tendencias en la investigación sobre efectividad, impacto y transferencia de la formación docente en educación superior. Educar, 51(1), 81-107. https://doi.org/10.5565/rev/educar.695

Fernández-Salinero Miguel, C., González Martín, M. R., y Belando Montoro, M. R. (2017). Mentoría pedagógica para profesorado universitario novel: estado de la cuestión y análisis de buenas prácticas. Estudios sobre Educación, 33, 49-75. https://doi.org/10.15581/004.33.49-75

Fink, L. D. (2013). Innovative Ways of Assessing Faculty Development. New Directions for Teaching and Learning, 133, 47-59. https://doi.org/10.1002/tl.20045

FUOC. (s. f.). Estatuts de la Fundació per a la Universitat Oberta de Catalunya. https://seuelectronica.uoc.edu/portal/_resources/CA/documents/seu-electronica/fuoc_estatuts_2014.pdf

Gaff, J., y Simpson, R. (1994). Faculty development in the United States. Innovative Higher Education, 18, 167-176. https://doi.org/10.1007/BF01191111

Generalitat de Catalunya. (s. f.). Universidades. http://universitats.gencat.cat/es/estudis_universitaris/universitats 
G. Hervas y J. L. Medina. Profesorado novel en las universidades catalanas: análisis de sus programas formativos

Generalitat de Catalunya. (2017). Flaix d'actuacions. Tercer trimestre 2017. Àrea de planificació i transparència. $\quad$ http://empresa.gencat.cat/web/.content/001-departament/04-serveis/01publicacions/Accio_govern/arxius/flaix_3r_trimestre_2017.pdf

Gimeno-Sacristán, J., y Pérez, A. I. (2002). Comprender y transformar la enseñanza. Morata.

Harland, T., y Staniforth, D. (2008). A family of strangers: The fragmented nature of academic development. Teaching in Higher Education, 13(6), 669-678. https://doi.org/10.1080/13562510802452392

Hsieh, H. F., y Shannon, S. E. (2005). Three Approaches to Qualitative Content Analysis. Qualitative Health Research, 15(9), 1277-1288. https://doi.org/10.1177/1049732305276687

Huberman, M. (1990). Las fases de la profesión docente. Ensayo de descripción y previsión. Qurriculum: Revista de Teoría, Investigación y Práctica Educativa, 2, 139-159. https://dialnet.unirioja.es/servlet/articulo?codigo=3607962

Imbernón, F. (2016). La formación del profesorado universitario: orientaciones y desorientaciones. Las prácticas de formación del profesorado universitario. En J. B. Martínez (coord.), Innovación en la universidad. Prácticas, políticas y retóricas (pp. 85-104). Graó.

Ingersoll, R. M., y Strong, M. (2011). The Impact of Induction and Mentoring Programs for Beginning Teachers. Review of Educational Research, 81(2), 201-233. https://doi.org/10.3102/0034654311403323

Jacob, W. J., Xiong, W., y Ye, H. (2015). Professional development programmes at world-class universities. Palgrave Communications, 1, 15002. https://doi.org/10.1057/palcomms.2015.2

Körkkö, M., Kyrö-Ämmälä, O., y Turunen, T. (2016). Professional development through reflection in teacher education. Teaching and Teacher Education, 55, 198-206. https://doi.org/10.1016/j.tate.2016.01.014

Krippendorff, K. (1989). Content analysis. En E. Barnouw, G. Gerbner, W. Schramm, T. L. Worth y L. Gross (eds.), International encyclopedia of communication (vol. 1, pp. 403-407). Oxford University Press.

Ley Orgánica 4/2007, de 12 de abril, por la que se modifica la Ley Orgánica 6/2001, de 21 de diciembre, de Universidades. https://www.boe.es/eli/es/lo/2007/04/12/4

Lortie, D. C. (1975). Schoolteacher: A sociological study. University of Chicago Press.

Luque-Vílchez, M. (2018). Los aprendizajes derivados de la participación en un plan de formación de profesorado universitario novel. International Journal of Educational Research and Innovation, 10, 153-162. https://www.upo.es/revistas/index.php/IJERI/article/view/2595/2728

Martín-Gutiérrez, Á., Conde-Jiménez, J., y Mayor-Ruiz, C. (2014). La identidad profesional docente del profesorado novel universitario. Revista de Docencia Universitaria, 12(4), 141-160. https://doi.org/10.4995/redu.2014.5618 
Mingorance Díaz, P., Mayor Ruiz, C., y Marcelo García, C. (1993). El primer año en la Universidad. Análisis de problemas de profesores principiantes. Revista de enseñanza universitaria, 5, 19-36. http://hdl.handle.net/11441/29300

Moll, R. (2018). La formación docente del profesorado universitario principiante en España: una mirada actual. En REDINE (ed.), Innovative strategies for Higher Education in Spain (pp. 112-122). Adaya Press.

Montes, D. A., y Suárez, C. I. (2016). La formación docente universitaria: claves formativas de universidades españolas. REDIE Revista Electrónica de Investigación Educativa, 18(3), 51-64. http://redie.uabc.mx/redie/article/view/996

Pagès Costas, T., Sayós Santigosa, R., Amador Campos, J. M., González Fernández, E., Marzo Ruiz, L., y Mato Ferré, M. (2016). Teacher training at the University of Barcelona: satisfaction, transfer and impact. @tic. Revista d'innovació educativa, 17, 41-48. https://doi.org/10.7203/attic.17.9103

Petty, N. J., Thomson, O. P., y Stew, G. (2012). Ready for a paradigm shift? Part 2: Introducing qualitative research methodologies and methods. Manual therapy, 17(5), 378-384. https://doi.org/10.1016/j.math.2012.03.004

Pinya, C. (2008). La formación permanente del profesorado universitario: estado de la cuestión. Revista Electrònica d'Investigació i Innovació Educativa i Socioeducativa, 1(0), 3-24. http://www.in.uib.cat/pags/volumenes/vol1_num0/pdfs/c_pinya.pdf

Porto Castro, A. M., y Mosteiro García, M. J. (2016). Calidad y formación del profesorado novel en las universidades públicas gallegas. Panorama, 10(9), 8-17. https://doi.org/10.15765/pnrm.v10i19.830

Sánchez Moreno, M., y Mayor Ruiz, C. (2006). Los jóvenes profesores universitarios y su formación pedagógica. Claves y controversias. Revista de educación, 339, 923-946. http://www.educacionyfp.gob.es/dam/jcr:1332d3d5-696a-402c-b47e-21e65f18bbf8/re33940pdf.pdf

Sorcinelli, M. D., Austin, A. E., Eddy, P. L., y Beach, A. L. (eds.) (2006). Creating the Future of Faculty Development - Learning from the Past, Understanding the Present. Anker.

Sorcinelli, M. D. (2007). Faculty Development: The Challenge Going Forward. Peer Review, 9(4), 4-9. https://www.aacu.org/publications-research/periodicals/faculty-development-challenge-goingforward

Steinert, Y. (2010). Becoming a better teacher: from intuition to intent. En T. Ende (ed.), Theory and practice of teaching medicine (pp. 73-93). American College of Physicians.

Torra Bitlloch, I., Màrquez Cebrian, M. D., Pagès Costas, T., Solà Ysuar, P., García Campos, R., Molina Luque, F., González Soto, À. P., y Sangrà Morer, A. (2013). Retos institucionales de la formación del profesorado universitario. Revista de Docencia Universitaria, 11(1), 285-309. https://doi.org/10.4995/redu.2013.5601 
G. Hervas y J. L. Medina. Profesorado novel en las universidades catalanas: análisis de sus programas formativos

Veenman, S. (1984). Perceived problems of beginning teachers. Review of Educational Research, 54(2), 143-178. https://doi.org/10.3102/00346543054002143

Zabalza Beraza, M. A. (2011). Evaluación de los planes de formación docente de las universidades. Educar, 47(1), 181-197. https://doi.org/10.5565/rev/educar.77

Zabalza Beraza, M. A., Cid Sabucedo, A., y Trillo Alonso, F. (2014). Formación docente del profesorado universitario. El difícil tránsito a los enfoques institucionales. Revista española de pedagogía, 72(257), 39-54. https://revistadepedagogia.org/wp-content/uploads/2014/02/257-03.pdf 